Pacific Journal of Mathematics

A NONASSOCIATIVE EXTENSION OF THE CLASS OF 


\title{
A NONASSOCIATIVE EXTENSION OF THE CLASS OF DISTRIBUTIVE LATTICES
}

\author{
E. FRIED AND G. GRÄTZER
}

Let $Z=\{0,1,2\}$ and define two binary operations $\wedge$ and $\vee$ on $Z$ as follows: $0 \wedge 1=0,0 \vee 1=1,1 \wedge 2=1,1 \vee 2=$ $2,2 \wedge 0=2,2 \vee 0=2$, both operations are idempotent and commutative. This paper deals with the equational class $Z$ generated by the algebra $\langle Z ; \wedge, \vee\rangle$. The class $Z$ contains the class of all distributive lattices and $Z$ is a subclass of the class of weakly associative lattices (trellis, $T$-lattice) in the sense of E. Fried and $H$. Skala.

The purpose of this paper is to prove that $Z$ shares the most important properties of the class of distributive lattices.

A tournament $\langle T ;\langle\rangle$ is a set $T$ with a binary relation $<$ such that for all $a, b \in T$ exactly one of $a=b, a<b$, and $b<a$ holds. Equivalently, a tournament is a directed graph without loops such that exactly one directed edge connects any two distinct points. Just as chains (linearly ordered sets) can be turned into lattices we can define meet and join on a tournament $\langle T ;\langle\rangle$ by the rule:

if $x<y$, then $x=x \wedge y=y \wedge x$ and $y=x \vee y=y \vee x$,

and $x=x \wedge x=x \vee x$ for all $x$.

Since for all $x, y \in T, x \neq y$, we have $x<y$ or $y<x$ the above rule defines $\wedge$ and $\vee$ on $T$.

Of course, the algebra $\langle T ; \wedge, \vee\rangle$ we constructed is not a lattice: neither $\wedge$ nor $\vee$ is associative unless $\langle T ;\langle\rangle$ is a chain, that is, $<$ is transitive. However, as it was observed in E. Fried [5], the two operations are idempotent, commutative; the absorption identities hold and also a weak form of the associative identities.

The smallest example of a nontransitive tournament is the threeelement cycle $\langle\{0,1,2\} ;<\rangle$ in which $0<1,1<2$, and $2<0$. In the corresponding algebra $Z$ neither $\wedge$ nor $\vee$ is associative.

$Z$ plays the same role for tournaments as the two-element lattice does for distributive lattices. A tournament (algebra) $\langle T ; \wedge, \vee\rangle$ is not a chain if and only if it contains $Z$ as a subalgebra.

In this paper we investigate the equational class $Z$ generated by the algebra $Z$. Observe that $C_{2}=\langle\{0,1\} ; \wedge, \vee\rangle$ is a subalgebra of $Z$, in fact, it is a two-element chain. Therefore, $Z$ contains as a subclass the class $\boldsymbol{D}$ of all distributive lattices. (Indeed, $\boldsymbol{D}$ is generated by $C_{2}$.)

The results of this paper can be summarized as follows: many of 
the most important properties of $\boldsymbol{D}$ generalize to $\boldsymbol{Z}$, and, in fact, $\boldsymbol{Z}$ is the only equational class (other than $\boldsymbol{D}$ ) generated by tournaments to which these results generalize.

In Part I, we discuss congruences in, and identities of $Z$. Section 2 contains some preliminary results and some important concepts, including the proper form of distributivity for tournaments. In $\S 3$ the minimal congruence relation $\Theta(a, b)$ is described in $Z$ and is applied to show that the Congruence Extension Property and the Amalgamation Property hold for $Z$. In $\S 4$ it is shown that the result of $\S 3$ characterizes the class $\boldsymbol{Z}$. This is applied in $\S 5$ to find a finite set of identities (in fact, two) characterizing the class $\boldsymbol{Z}^{1}$. Part II contains the structure theorems. In $\S 6$ we describe the structure of finite algebras in $Z$ : they are all of the form $D \times Z^{k}$, where $D$ is a uniquely determined distributive lattice. Section 7 gives the structure of free algebras over $Z$ : the free algebra on $n$ generators is of the form $F_{D}(n) \times Z^{k_{n}}$, where $F_{D}(n)$ is the free distributive lattice on $n$ generators and $k_{n}=3^{n-1}-2^{n}+1$. We prove in $\S 8$ that every algebra in $Z$ can be embedded in an injective one. The injectives in $\boldsymbol{D}$ are known to be the complete Boolean lattices. The injectives in $Z$ are the extensions of $Z$ by complete Boolean lattices.

ExAmples. An "evaluation" of elements of a set $A$ is a map $\varphi$ of $A$ into another set $S$, equipped with a binary relation $<$, meaning "better than". We say that $b$ is better than $a(a, b \in A)$ if $a \varphi<b \varphi$. If we want to be able to compare any two elements of $A$, then we have to assume that $\langle S ;\langle\rangle$ is a tournament.

Evaluating a sample $\left\langle a_{1}, \cdots, a_{n}\right\rangle$ of elements of $A$ we get an "evaluation vector": $\left\langle a_{1} \varphi, \cdots, a_{n} \varphi\right\rangle$. The study of the equational class generated by $\langle S ; \wedge, \vee\rangle$ is the investigation of the algebra of the evaluation vectors. Thus $Z$ is the "algebra" of the evaluation vectors over $Z$.

Given a set $X$ we can consider the set $P(X)$ of all partitions $\left\langle X_{0}, X_{1}\right\rangle$ of $X$ into two sets. If $\left\langle X_{0}, X_{1}\right\rangle,\left\langle Y_{0}, Y_{1}\right\rangle \in P(X)$ we can set $\left\langle X_{0}, X_{1}\right\rangle \leqq\left\langle Y_{0}, Y_{1}\right\rangle$ if and only if $X_{0} \subseteq Y_{0}$. This makes $P(X)$ into a distributive lattice. Any distributive lattice is a sublattice (up to isomorphism) of some $P(X)$.

Now consider the set $Z(X)$ of all partitions of $X$ into three subsets $\left\langle X_{0}, X_{1}, X_{2}\right\rangle$. For $\left\langle X_{0}, X_{1}, X_{2}\right\rangle,\left\langle Y_{0}, Y_{1}, Y_{2}\right\rangle \in Z(X)$ we declare $\left\langle X_{0}, X_{1}\right.$,

1 The results of this paper were announced in the Notices of the American Mathematical Society 18 (1971), 402 and 548. Independently, in $1971 \mathrm{~K}$. Baker announced in a lecture a general result, namely that every equational class of finite type in which the algebras have distributive congruence lattices and which is generated by a finite algebra can be defined by a finite set of identities. Our result in $\S 5$ is a very special case of Baker's result. Of course, the general method of Baker yields more complicated identities for $\boldsymbol{Z}$. 
$\left.X_{2}\right\rangle \leqq\left\langle Y_{0}, Y_{1}, Y_{2}\right\rangle$ if and only if $X_{0} \subseteq Y_{0} \cup Y_{1}, X_{1} \subseteq Y_{1} \cup Y_{2}$, and $X_{2} \subseteq$ $Y_{2} \cup Y_{0}$. Again, $Z(X) \in Z$, and any member of $Z$ will be (up to isomorphism) a subalgebra of some $Z(X)$. Observe that $Z(X)$ contains $P(X)$ as a subalgebra under the correspondence $\left\langle X_{0}, X_{1}\right\rangle \rightarrow\left\langle X_{1}, X_{0}, \varnothing\right\rangle$.

PART I. Congruences and Identities.

2. Preliminary results. An algebra $\langle A ; \wedge, \vee\rangle$ is called a weakly associative lattice (WA-lattice) if it satisfies the following set of identities

$$
\begin{array}{ll}
x \wedge x=x \quad \text { and } \quad x \vee x=x & \text { (idempotency); } \\
x \wedge y=y \wedge x \text { and } x \vee y=y \vee x \quad \text { (commutativity); } & \\
x \wedge(x \vee y)=x \quad \text { and } x \vee(x \wedge y)=x &
\end{array}
$$

(absorption identities) ;

$$
\begin{aligned}
& ((x \wedge z) \vee(y \wedge z)) \vee z=z \quad \text { and } \\
& ((x \vee z) \wedge(y \vee z)) \wedge z=z \quad \text { (weak associativity) }
\end{aligned}
$$

This axiom system was discovered independently by E. Fried [5] (he called these T-lattices) and H. M. Skala [16] (she called them trellis).

(1)-(4) are not independent. (3) implies (1), and (4) and (1) imply (3). Observe, that the first identity (and, similarly, the second identity) of (4) can be written in the form

$$
((x \wedge z) \vee(y \wedge z)) \vee z=(x \wedge z) \vee((y \wedge z) \vee z)
$$

which justifies the name weak associativity.

It is easy to see that in a $W A$-lattice the polynomial $p(x, y, z)=$ $((x \wedge y) \vee(y \wedge z)) \vee(z \wedge x)$ satisfies the identities

$$
x=p(x, x, y)=p(x, y, x)=p(y, x, x)
$$

implying (B. Jónsson [13]) that

Lemma 1. The congruence lattice of a WA-lattice is distributive.

If $A$ and $B$ are $W A$-lattices, $\Theta$ a congruence relation of $A, \Phi$ a congruence relation of $B$, then we can define a congruence relation $\Theta \times \Phi$ on $A \times B:\langle a, b\rangle \equiv\left\langle a_{1}, b_{1}\right\rangle(\Theta \times \Phi)$ if $a \equiv a_{1}(\Theta)$ and $b \equiv b_{1}(\Phi)$. Let $C(D)$ denote the congruence lattice of $D$. Lemma 1 is known to imply

CoROLLARY. Every congruence relation of $A \times B$ is of the form 
$\Theta \times \Phi$ with $\Theta \in C(A)$ and $\Phi \in C(B)$. Therefore, $C(A \times B) \cong C(A) \times C(B)$.

Combining Lemma 1 with another result of B. Jónsson [13] we get the crucial

Lemma 2. Let $A$ be a finite $W A$-lattice and let $K$ be the equational class generated by $A$. Then every algebra in $K$ is isomorphic to a subalgebra of an algebra of the form $A_{1}^{I_{1}} \times \cdots \times A_{n}^{I_{n}}$, where $I, \cdots, I_{n}$ are arbitrary sets and $A_{1}, \cdots, A_{n}$ are homomorphic images of subalgebras of $A$.

In a $W A$-lattice $A$ we can define

$$
x \leqq y \text { if and only if } x=x \wedge y \text { if and only if } y=x \vee y \text {. }
$$

The equivalence of the second and third clauses follows from the absorption identities. Observe that $x \leqq x$, and $x \leqq y$ and $y \leqq x$ imply $x=y$. Also, $x \leqq x \vee y, y \leqq x \vee y$, and it follows from (4) that $x \leqq t$ and $y \leqq t$ imply $x \vee y \leqq t$; these can be summarized by stating that $x \vee y$ is the least upper bound of $x$ and $y$. Dually, $x \wedge y$ is the greatest lower bound of $x$ and $y$. These properties give an alternative definition of $W A$-lattices in terms of $\leqq(E$. Fried [5] and M. H. Skala [16]).

We conclude from this immediately, that any tournament is a $W A$-lattice. Furthermore, since a homomorphic image of a tournament $A$ is isomorphic to a subalgebra of $A$ we conclude from Lemma 2:

Lemma 3. Let $A$ be a finite tournament. Then the equational class $K$ generated by $A$ consists of subalgebras of direct powers of $A$. In particular, every subdirectly irreducible member of $K$ is a subalgebra of $A$.

Applying this to $Z$ and to the equational class $Z$ it generates we conclude that every member of $Z$ is isomorphic to a subalgebra of some $Z^{I}$. The subdirectly irreducible algebras in $Z$ are $Z$ and $C_{2}$. Thus $\boldsymbol{Z}$ contains $\boldsymbol{D}$, in fact, $\boldsymbol{Z}$ covers $\boldsymbol{D}$.

Given an algebra $A$ and $a, b \in A$ there is a smallest congruence relation $\Theta$ under which $a \equiv b(\Theta)$. This congruence relation is denoted by $\Theta(a, b)$; it is called a principal congruence relation. Principal congruences of distributive lattices are described in G. Grätzer and E. T. Schmidt [10] and G. Grätzer [8]:

LEMma 4. Let $L$ be a distributive lattice, $a, b, c, d \in L, a \leqq b$, and $c \leqq d$. Then the following conditions are equivalent:

(i ) $c \equiv d(\Theta(a, b))$; 
(ii) $c=(a \vee c) \wedge d$ and $d=(b \vee c) \wedge d$.

(iii) $a \wedge c=a \wedge d$ and $b \vee c=b \vee d$.

The most important result of this paper, namely Theorem 2, is patterned after Lemma 4.

Lemma 4 implies that any distributive lattice $L$ has the property that $c \equiv d(\Theta(a, b))$ can be decided in the sublattice generated by $a, b$, $c$, and $d$. This property has an important consequence by A. Day [4]:

LemMa 5. Let $\boldsymbol{K}$ be an equational class of algebras with the property that for any $A \in \boldsymbol{K}$ and $a, b, c, d \in A, c \equiv d(\Theta(a, b))$ can be decided in the subalgebra generated by $a, b, c$, and $d$. Then $\boldsymbol{K}$ has the Congruence Extension Property, that is, if $A, B \in \boldsymbol{K}, A$ a subalgebra of $B$ and if $\Theta$ is a congruence relation on $A$, then there is a congruence relation $\Phi$ on $B$ such that $\Phi$ restricted to $A$ is $\Theta$.

Another property of distributive lattices we need to generalize is the uniqueness of relative complements.

Let $\boldsymbol{T}$ denote the equation class generated by all tournaments.

Lemma 6. The distributive law

$$
x \wedge(y \vee z)=((x \wedge y) \vee(x \wedge z)) \wedge(y \vee z)
$$

holds in $\boldsymbol{T}$.

Proof. Let $A$ be a tournament, $x, y, z \in A$. If two of $x, y$, and $z$ are equal, then (5) holds since it is true in lattices. If $\{x, y, z\}$ is a chain, again, (5) is trivial. So we can assume that $\{x, y, z\}$ is isomorphic to $Z$. Since (5) is symmetric in $y$ and $z$, we can assume that $y<z$. Therefore, $y<z<x<y$. In this case, $x \wedge(y \vee z)=$ $x \wedge z=z$ and $((x \wedge y) \vee(x \wedge z)) \wedge(y \vee z)=(x \vee z) \wedge z=z$, so (5) holds. Thus (5) holds for all algebras generating $\boldsymbol{T}$, so it holds for $\boldsymbol{T}$.

Lemma 7. Let $A$ be a WA-lattice satisfying (5). Then for $a, b$, $c \in A, a \wedge b=a \wedge c$ and $a \vee b=a \vee c$ imply that $b=c$.

\section{Proof.}

$$
\begin{aligned}
b & =b \wedge(a \vee b) \\
& =b \wedge(a \vee c) \\
& =((b \wedge a) \vee(b \wedge c)) \wedge(a \vee c) \\
& =((c \wedge a) \vee(b \wedge c)) \wedge(a \vee b) \\
& =c \wedge(a \vee b) \\
& =c \wedge(a \vee c) \\
& =c
\end{aligned}
$$
since $a \vee b=a \vee c$

since $b \wedge a=c \wedge a$ and $a \vee c=a \vee b$ by (5) since $a \vee b=a \vee c$ by (3), 
which was to be proved.

It should be pointed out that, unlike in lattices, (5) is not selfdual. The independence of (5) and its dual is shown in [6].

In conclusion we mention that a list of identities describing $\boldsymbol{T}$ was given in [9].

3. Principal congruences. In this section we state and verify the analogue of Lemma 4 for $Z$. To facilitate the discussion we introduce some notation. We define five polynomials in the variables $x_{1}, x_{2}, x_{3}, x_{4}$, and $x_{5}$ :

$$
\begin{gathered}
p_{1}=x_{2} \wedge x_{5}, p_{2}=x_{2} \vee x_{4}, p_{3}=\left(x_{3} \vee x_{5}\right) \vee x_{4}, \text { and } p_{4}=x_{5} \\
p=\left(\left(\left(x_{1} \vee p_{1}\right) \vee p_{2}\right) \wedge p_{3}\right) \wedge p_{4} .
\end{gathered}
$$

Theorem 1. Let $A \in \boldsymbol{Z}$, and let $a, b, c, d \in A, a \leqq b$, and $c \leqq d$. Then the following conditions are equivalent:

(i ) $c \equiv d(\Theta(a, b))$;

(ii) $c=p(a, a, b, c, d)$ and $d=p(b, a, b, c, d)$;

(iii) $a \wedge(c \wedge b)=a \wedge(d \wedge b)$ and $(a \vee c) \vee b=(a \vee d) \vee b$.

REMARK. If $A$ is a lattice, then $p_{1} \leqq x_{2} \leqq p_{2}$; similarly, $p_{3} \geqq$ $x_{5}=p_{4}$. Therefore, $p=\left(x_{1} \vee\left(p_{1} \vee p_{2}\right)\right) \wedge\left(p_{3} \wedge p_{4}\right)=\left(x_{1} \vee p_{2}\right) \wedge x_{5}=$ $\left(x_{1} \vee\left(x_{2} \wedge x_{5}\right)\right) \wedge x_{5}$, reducing the first half of (ii) to the first half of Lemma 4. (ii). The second half of (ii) can be handled similarly. As for (ii), $\wedge$ and $\vee$ are associative if $A$ is a lattice, and so $\alpha \wedge(c \wedge b)=$ $b \wedge c, a \wedge(d \wedge b)=a \wedge d$, and so on, yielding Lemma 4. (iii). Observe the different placing of the parentheses in the two equations in (iii).

Proof. (i) implies (ii). We prove this implication in several steps.

( $\alpha) \quad A$ is isomorphic to $C_{2}=\{0,1\}$. Since $C_{2}$ is a lattice a reference to Lemma 4 settles the matter. Or, equivalently, check the implication for $a=0, b=1$ and $c=0, d=1$, or $c=d=0$, or $c=d=1$, and for $a=b=1$ and $c=d=0$ or $c=d=1$ (seven cases).

( $\beta$ ) $A$ is (isomorphic to) $Z=\{0,1,2\}$. If $\{a, b, c, d\} \neq Z$, then we proceed as under $(\alpha)$. If $a=b$, then we must have $c=d$, thus we can assume that $a \neq b$. By symmetry, we can assume that $a=0, b=$ 1. Since $Z$ is simple, $\langle c, d\rangle$ could be $\langle 2,2\rangle,\langle 1,2\rangle$, or $\langle 2,0\rangle$ (all other pairs contradict that $c \leqq d$ or that $\{a, b, c, d\}=Z)$. Therefore, it is sufficient to check the implication in $Z^{3}$ for $a=\langle 0,0,0\rangle, b=\langle 1,1,1\rangle$, $c=\langle 2,1,2\rangle$, and $d=\langle 2,2,0\rangle$. Compute: 


$$
\begin{aligned}
p_{1} & =a \wedge d=\langle 2,2,0\rangle, p_{2}=a \vee c=\langle 0,1,0\rangle, p_{3}=(b \vee d) \vee c \\
& =\langle 2,2,1\rangle \vee\langle 2,1,2\rangle=\langle 2,2,2\rangle, p_{4}=\langle 2,2,0\rangle, p(a, a, b, c, d) \\
& =\left(\left(\left(a \vee p_{1}\right) \vee p_{2}\right) \wedge p_{3}\right) \wedge p_{4} \\
& =(((\langle 0,0,0\rangle \vee\langle 2,2,0\rangle) \vee\langle 0,1,0\rangle) \wedge\langle 2,2,2\rangle) \wedge\langle 2,2,0\rangle \\
& =((\langle 0,0,0\rangle \vee\langle 0,1,0\rangle) \wedge\langle 2,2,2\rangle) \wedge\langle 2,2,0\rangle \\
& =(\langle 0,1,0\rangle \wedge\langle 2,2,2\rangle) \wedge\langle 2,2,0\rangle \\
& =\langle 2,1,2\rangle \wedge\langle 2,2,0\rangle=\langle 2,1,2\rangle=c,
\end{aligned}
$$

and similarly, $p(b, a, b, c, d)=d$.

(y) Assume the implication to hold for the algebras $A_{1}, \cdots, A_{n}$, and let $B$ be a subalgebra of $A_{1} \times \cdots \times A_{n}$. Then the implication holds in $B$. Indeed, let $a, b, c, d \in B, a \leqq b, c \leqq d$ and let $c \equiv d(\Theta(a, b))$ in $B$. By a result of A. I. Malcev (see Theorem 10.3 of [7]) there is a sequence of elements $z_{0}=c, z_{1}, \cdots, z_{m}=d$ of $B$, and unary algebraic functions $p_{0}, \cdots, p_{n-1}$ of $B$ such that $\left\{p_{i}(a), p_{i}(b)\right\}=\left\{z_{i}, z_{i+1}\right\}$ for $i=$ $0,1, \cdots, m-1$.

For an element $u$ of $B$ let $u^{(i)}$ denote the $i$ th component of $u$, that is, $u=\left\langle u^{(i)}, \cdots, u^{(n)}\right\rangle$. A unary algebraic function is of the form $q\left(u_{1}, \cdots, x, \cdots, u_{k}\right)$, where $u_{1}, \cdots, u_{k} \in B$ and $p$ is a polynomial. So we can define $p^{(i)}$ a unary algebraic function on $A_{i}$ by $q\left(u_{1}^{(i)}, \cdots, x\right.$, $\left.\cdots, u_{k}^{(i)}\right)$.

Using the sequence of elements of $A_{i}: z_{0}^{(i)}, z_{1}^{(i)}, \cdots, z_{m}^{(i)}$ of $B_{i}$, and the unary algebraic functions: $p_{0}^{(i)}, \cdots, p_{n-1}^{(i)}$, we conclude that

$$
a^{(i)} \equiv b^{(i)}\left(\Theta\left(c^{(i)}, d^{(i)}\right)\right) \text { in } A_{i} \text {. }
$$

Thus, by assumption,

$$
\begin{aligned}
& c^{(i)}=p\left(a^{(i)}, a^{(i)}, b^{(i)}, c^{(i)}, d^{(i)}\right) \text { and } \\
& d^{(i)}=p\left(b^{(i)}, a^{(i)}, b^{(i)}, c^{(i)}, d^{(i)}\right),
\end{aligned}
$$

for $i=1, \cdots, n$. Hence, $c=p(a, a, b, c, d)$ and $d=p(b, a, b, c, d)$, which was to be proved.

Now we are ready to prove the implication. Let $A \in Z, a, b, c, d \in$ $A, a \leqq b, c \leqq d$, and $c \equiv d(\Theta(a, b))$. Invoking Malcev's result used above we can assume that $A$ is finitely generated. Since $Z$ is generated by $Z$, an $n$-generated algebra can have no more than $3^{3^{n}}$ elements, hence it is finite. Thus $A$ is finite. By Lemma $3, A$ can be embedded in some $Z^{k}$. By $(\alpha)$ and $(\beta)$ the implication holds in $Z$, hence by $(\gamma)$ it holds in $A$, completing the proof.

(ii) implies (iii). This implication takes the form of a universal Horn sentence (see, for instance, [7], §46), therefore, it holds in $Z$ if and only if it holds in $Z$. In $Z$, if $a=b$, then the assumption implies 
$c=d$, hence the conclusion obviously holds. If $a \neq b(a<b)$, then the functions $a \wedge(x \wedge b)$ and $(a \vee x) \vee b$ are constants $(a \wedge(x \wedge b)=a$ and $(a \vee x) \vee b=b)$, so the conclusion is obvious.

(iii) implies (i). Let $\Theta=\Theta(a, b)$ and let $[x] \Theta$ denote the congruence class of $A$ containing $x$. Then

$$
[a \wedge(c \wedge b)] \Theta=[a \wedge(d \wedge b)] \Theta,
$$

and so, using $[a] \Theta=[b] \Theta$, and $x \wedge(y \wedge x)=y \wedge x$, we obtain, in turn:

$$
\begin{gathered}
{[a] \Theta \wedge([c] \Theta \wedge[b] \Theta)=[a] \Theta \wedge([d] \Theta \wedge[b] \Theta)} \\
{[a] \Theta \wedge[c] \Theta=[a] \Theta \wedge[d] \Theta .}
\end{gathered}
$$

Similarly,

$$
[a] \Theta \vee[c] \Theta=[a] \Theta \vee[d] \Theta .
$$

Applying Lemma 7 to $A / \Theta,(8)$ and (9) imply that $[c] \Theta=[d] \Theta$, that is, $c \equiv d(\Theta(a, b))$, completing the proof of Theorem 1 .

Observe, that Theorem 1 implies that the assumptions of Lemma 5 are satisfied in $Z$. Thus,

\section{CoRollary 1. $\boldsymbol{Z}$ has the Congruence Extension Property.}

A class $K$ of algebras is said to have the Amalgamation Property if for any $A, B_{1}, B_{2} \in K$, and embeddings $f_{i}: A \rightarrow B_{i}, i=1,2$, there is a $C \in K$ and embeddings $g_{i}: B_{i} \rightarrow C, i=1,2$, such that $f_{1} g_{1}=f_{2} g_{2}$. For a general discussion of the Amalgamation Property see B. Jónsson [12].

\section{Corollary 2. $\boldsymbol{Z}$ has the Amalgamation Property.}

Proof. By Theorem 13.16 of [8] it is sufficient to prove that for given $A, B_{1}, B_{2} \in Z$, embeddings $f_{i}: A \rightarrow B_{i}, i=1,2$, and $a, b \in B_{1}, a \neq$ $b$, there exist homomorphisms $g_{i}: B_{i} \rightarrow Z, i=1,2$, such that $f_{1} g_{1}=f_{2} g_{2}$ and $a g_{1} \neq b g_{1}$. By Lemma 3 , there is a homomorphism $g_{1}: B_{1} \rightarrow Z$ satisfying $a g_{1}=b g_{1}$. Let $\Theta$ be the congruence relation of $A$ induced by $g_{1}$. By Corollary 1 , there exists a congruence relation $\bar{\Theta}$ on $B_{2}$ satisfying $\bar{\Theta}_{A}=\Theta$. Let $g_{2}^{\prime}$ be the natural homomorphism of $B_{2}$ onto $B_{2} / \bar{\Theta}$. By Lemma 3 again, there is a homomorphism $g_{2}^{\prime \prime}: B_{2} / \bar{\Theta} \rightarrow Z$. We define $g_{2}=g_{2}^{\prime} g_{2}^{\prime \prime}$. Obviously, $f_{1} g_{1}=f_{2} g_{2}$, concluding the proof.

In closing this section, we mention that the polynomial $p$, which plays a central role in Theorem 1, was found using free algebras. A free algebra was used also to discover the identity (5) in order to get Lemma 7.

Alternate forms of $p$ such as $p=\left(\left(\left(x_{1} \vee q_{1}\right) \wedge q_{2}\right) \vee q_{3}\right) \wedge q_{4}$ or any 
of the other possibilities can also be found using the free algebra technique.

4. The characterization theorem. We started out in our research trying to find a finite set of identities characterizing $Z$. Since we believed that the equivalence of Lemma 4 . (i) and (ii) is characteristic of $\boldsymbol{D}$ we wanted to find the analogous result for $\boldsymbol{Z}$ hoping that it would characterize $Z$. The next step would then be to find a set of identities based on which the analogous result for $\boldsymbol{Z}$ can be proved. As we shall see in $\S 5$, this runs into some problems. The situation was saved by Theorem 1. (iii) and by the fact that Theorem 1. (iii) can also be used to characterize $Z$. Since this is the result needed in $\S 5$ we omit the original theorem and prove only the latter one.

THEOREM 2. Let $\boldsymbol{K}$ be an equational class of WA-lattices in which for any $A \in \boldsymbol{K}, a, b, c, d \in A, a \leqq b, c \leqq d$, and $c \equiv d(\Theta(a, b))$ imply that $a \wedge(c \wedge b)=a \wedge(d \wedge b)$ and $(a \vee c) \vee b=(a \vee d) \vee b$. Then $\boldsymbol{K} \leqq Z$.

Let $A$ be a subdirectly irreducible algebra in $\boldsymbol{K}$. We shall prove that $A \cong C_{2}$ or $A \cong Z$. This obviously implies that $K \cong Z$.

If $|A|=2$, then $A \cong C_{2}$ since $A$ is a $W A$-lattice. Thus we can assume that $|A|>2$.

Since $A$ is a subdirectly irreducible algebra with more than two elements, $A$ has a congruence relation $\Phi \neq \omega$ with the property that $\Phi \leqq \Theta$ for any congruence relation $\Theta$ of $A$ with $\Theta \neq \omega$. Since $\Phi \neq \omega$ there is a congruence class $G$ of $\Phi$ of more than one element.

We claim that there are elements $a, b, c \in A$ such that $a, b \in G$, $a<b$, and $c<a$ or $b<c$. To prove this take $x, y \in G, x \neq y$. Obviously, $x \wedge y \in G$ and $x \neq x \wedge y$ or $y \neq x \wedge y$. Set $a^{\prime}=x \wedge y$ and $b^{\prime}=x$ or $b^{\prime}=y$ so that $a^{\prime} \neq b^{\prime}$. If $a^{\prime}$ and $b^{\prime}$ do not satisfy the requirements with some $c^{\prime} \in A$, then for all $d \in A$ we have $a^{\prime} \wedge d=a^{\prime}$ and $b^{\prime} \vee d=b^{\prime}$, that is, $a^{\prime}<d<b^{\prime}$ for any $d \in A, d \neq a^{\prime}, d \neq b^{\prime}$. In this case set $c=a^{\prime}, a=d$, and $b=b^{\prime}$.

So we can assume that we have

$$
c<a<b, a, b \in G,
$$

since the other case, $a<b<c$ can be proceeded with similarly (dually).

By (10) $c \neq a$, so $\Theta(c, a) \neq \omega$. By the definition of $\Phi$, we have $\Phi \leqq \Theta(c, a)$, and by the definition of $G$ and (10) we must have

$$
a \equiv b(\Theta(c, a)) \text {. }
$$

We apply the hypothesis of Theorem 2 to this congruence. We obtain:

$$
(c \vee a) \vee a=(c \vee b) \vee a,
$$


that is, $a=(c \vee b) \vee a$, in other words,

$$
c<b \leqq a .
$$

We claim that

$$
c=c \vee b .
$$

Assume, to the contrary, that $c<c \vee b$. Then $\Theta(c, c \vee b) \neq \omega$, and so as above we get

$$
a \equiv b(\Theta(c, c \vee b))
$$

Applying the hypothesis of Theorem 2 to this congruence we obtain:

$$
c \wedge(a \wedge(c \vee b))=c \wedge(b \wedge(c \vee b)) .
$$

By (11), $a \wedge(c \vee b)=a$ and, by (10), $c \wedge a=c$ so (13) yields $c=$ $c \wedge b$, or, equivalently,

$$
c \leqq b .
$$

On the other hand, by (11), $c \vee b \leqq a$; combining this with (14) we obtain $b \leqq a$, contradicting (10). This verifies (12).

(10) and (12) jointly mean that $\{a, b, c\}$ is a subalgebra of $A$ and $\{a, b, c\}$ is isomorphic to $Z$.

We claim that $A=\{a, b, c\}$.

Assume to the contrary that there is an element $d \in A$ such that $d \notin\{a, b, c\}$. We claim that $d$ can be chosen to be comparable to one of $a, b$, and $c$. Indeed, if there is no such $d$ then for an arbitrary $e \in A, a \wedge e=a$, since $a \wedge e<a$ implies that $a \wedge e=c$ and so $c<e$. Similarly, $a \vee e=a$, implying that $a=e$, a contradiction. Thus, by reason of symmetry and duality we can assume that there is an element $d \in A$ satisfying

$$
d \in\{a, b, c\} \text { and } d<a .
$$

Since $a, b \in G$ and $b<c<a$ we conclude that $c \in G$. Thus $d \neq a$ implies the congruence

$$
b \equiv c(\Theta(d, a))
$$

Therefore,

$$
d \wedge(b \wedge a)=d \wedge(c \wedge a) \text {. }
$$

But by (10) $b \wedge a=a$ and $c \wedge a=c$; by (15), $d \wedge a=d$, hence (16) yields: $d=d \wedge c$. Since $c \neq d$ this means that $d<c$. So we get the congruence

$$
a \equiv b(\Theta(d, c))
$$


which implies that

$$
(d \vee a) \vee c=(d \vee b) \vee c .
$$

But $d \vee a=a, a \vee c=c$, hence (17) gives

$$
a=(d \vee b) \vee c .
$$

Observe that $d \leqq c$ and $b \leqq c$. Therefore, $d \vee b \leqq c$, and so $(d \vee b) \vee c$ $=c$, contradicting (18) and $a \neq c$.

This contradiction shows that $A=\{a, b, c\}$, that is, $A \cong Z$, which completes the proof of Theorem 2 .

5. Identities for $Z$. We want to find a finite set $\Sigma$ of identities characterizing $Z$. This set $\Sigma$ should express that $Z$ is a class of $W A$-lattices in which minimal congruences can be described by Theorem 1. It is easy to find identities which imply that the relation given by Theorem 1. (ii) is reflexive, symmetric, and has the Substitution Property for $\wedge$ and $\vee$. However, transitivity takes the form "if Theorem 1. (ii) holds for $c, d$, and for $c_{1}, d_{1}$, and $d=c_{1}$, then it holds for $c, d_{1}$ " which we could not turn into an identity.

The trick is to find identities that prove that $\Theta(a, b)$ is in some sense the transitive extension of the relation given by Theorem 1 . (ii). Then to show that this implies that Theorem 1. (iii) can be used to describe $\Theta(a, b)$.

We need some notation. We shall use $p, p_{1}, \cdots, p_{4}$ of (6) and (7) without references. Two 4-ary polynomials derived from $p$ will be used often:

$$
\begin{gathered}
q_{1}=p\left(x_{1}, x_{1}, x_{1} \vee x_{2}, x_{3}, x_{3} \vee x_{4}\right) \\
q_{2}=p\left(x_{1} \vee x_{2}, x_{1}, x_{1} \vee x_{2}, x_{3}, x_{3} \vee x_{4}\right) .
\end{gathered}
$$

Finally, for the polynomials $t_{1}, t_{2}, t_{3}$, and $t_{4}$, let $R\left(t_{1}, t_{2}, t_{3}, t_{4}\right)$ denote the identities

$$
t_{3}=q_{1}\left(t_{1}, t_{2}, t_{3}, t_{4}\right) \quad \text { and } \quad t_{3} \vee t_{4}=q_{2}\left(t_{1}, t_{2}, t_{3}, t_{3} \vee t_{4}\right)
$$

$\Sigma$ consists of three sets of identities. $\Sigma_{1}$ is a set of identities for $W A$-lattices (for instance, (1)-(4)) and one more identity

$$
((x \vee y) \vee(x \wedge z)) \vee(x \vee z)=(x \vee(y \vee z)) \vee z .
$$

$\Sigma_{2}$ is the following eight identities:

$$
\begin{gathered}
R\left(x_{1}, x_{2}, q_{i} \wedge x_{5}, q_{j} \wedge x_{5}\right),\{i, j\}=\{1,2\} \\
R\left(x_{1}, x_{2}, q_{i} \vee x_{5}, q_{j} \vee x_{5}\right),\{i, j\}=\{1,2\} .
\end{gathered}
$$


$\Sigma_{3}$ consists of two identities

$$
\begin{aligned}
& x_{1} \wedge\left(q_{1} \wedge\left(x_{1} \vee x_{2}\right)\right)=x_{1} \wedge\left(q_{2} \wedge\left(x_{1} \vee x_{2}\right)\right) \\
& \left(x_{1} \vee q_{1}\right) \vee\left(x_{1} \vee x_{2}\right)=\left(x_{1} \vee q_{2}\right) \vee\left(x_{1} \vee x_{2}\right)
\end{aligned}
$$

THeOREM 3. $\Sigma=\Sigma_{1} \cup \Sigma_{2} \cup \Sigma_{3}$ defines the class $Z$.

Proof. First we have to see that $\Sigma$ is satisfied in $\boldsymbol{Z} . \Sigma_{1}$ is obviously satisfied excepting (22).

It is sufficient to verify (22) in $Z$. Let $a, b, c \in Z$. If $|\{a, b, c\}| \leqq$ 2 , then they form a sublattice, in which (22) becomes $(a \vee b) \vee$ $(a \vee c)=a \vee(b \vee c)$, a triviality. Thus we can assume that $Z=$ $\{a, b, c\}$. If $a=0, b=1, c=2$, then $((0 \vee 1) \vee(0 \wedge 2)) \vee(0 \vee 2)=$ $(1 \vee 2) \vee 0=2 \vee 0=0$ and $((2 \vee 1) \vee 0) \vee 2=(2 \vee 0) \vee 2=0 \vee 2=0$; if $a=1, b=0, c=2$, then $((1 \vee 0) \vee(1 \wedge 2)) \vee(1 \vee 2)=((1 \vee 1) \vee 1) \vee 2=$ $1 \vee 2=2$ and $((2 \vee 0) \vee 1) \vee 2=(0 \vee 1) \vee 2=1 \vee 2=2$. All the other substitutions agree with one of these two (up to automorphism) showing (22) in $Z$. If $x_{1} \equiv x_{1} \vee x_{2}$, then by (19), and (20), $q_{1} \equiv q_{2}$ and so $q_{1} \wedge x_{5} \equiv q_{2} \wedge x_{5}$. In other words,

$$
q_{1} \wedge x_{5} \equiv q_{2} \wedge x_{5}\left(\Theta\left(x_{1}, x_{1} \vee x_{2}\right)\right)
$$

or

$$
q_{1} \wedge x_{5} \equiv\left(q_{1} \wedge x_{5}\right) \vee\left(q_{2} \wedge x_{5}\right)\left(\Theta\left(x_{1}, x_{1} \vee x_{2}\right)\right) .
$$

Applying Theorem 1. (ii) to this congruence we obtain

$$
\begin{aligned}
q_{1} \wedge x_{5}=q_{1}\left(x_{1}, x_{1} \vee x_{2}, q_{1} \wedge x_{5},\left(q_{1} \wedge x_{5}\right) \vee\left(q_{2} \wedge x_{5}\right)\right) \\
\left(q_{1} \wedge x_{5}\right) \vee\left(q_{2} \wedge x_{5}\right)=q_{2}\left(x_{1}, x_{1} \vee x_{2}, q_{1} \wedge x_{5},\left(q_{1} \wedge x_{5}\right) \vee\left(q_{2} \wedge x_{5}\right)\right) .
\end{aligned}
$$

By (21), these two are written in the form $R\left(x_{1}, x_{1} \vee x_{2}, q_{1} \wedge x_{5}, q_{2} \wedge x_{5}\right)$. The other six identities under (23) and (24) are similarly proved. Finally, since $q_{1} \equiv q_{2}\left(\Theta\left(x_{1}, x_{1} \vee x_{2}\right)\right)$, an application of Theorem 1. (iii) proves (25) and (26).

Now let $K$ be the class of all algebras satisfying $\Sigma$. By what we have proved above, $Z \subseteq K$.

Let $A \in K, a, b \in A$, and $a \leqq b$. We define a binary relation $\Phi$ on $A$ :

$c \equiv d(\Phi)$ if and only if there exists a sequence $c=r_{0}, r_{1}, \cdots, r_{n}=d$ of elements of A such that, for all $i=0, \cdots, n-1, r_{i}$ and $r_{i+1}$ are comparable and $R\left(a, b, r_{i} \wedge r_{i+1}, r_{i} \vee r_{i+1}\right)$.

We claim that $\Phi$ is a congruence relation, in fact, $\Phi=\Theta(a, b)$.

$\Phi$ is obviously symmetric and transitive. Next we show that $\Phi$ is reflexive, in other words, for all $c \in A, R(a, b, c, c)$. By (19)-(21), this means that 
(27)

$$
p(a, a, b, c, c)=p(b, a, b, c, c)=c .
$$

Using (6) we compute: $p_{1}=a \wedge c, p_{2}=a \vee c, p_{3}=(b \vee c) \vee c=b \vee c$ (by $(3)), p_{4}=c$, and so

$$
\begin{array}{rlrl}
p(a, a, b, c, c) & =(((a \vee(a \wedge c)) \vee(a \vee c)) \wedge(b \vee c)) \wedge c \text { by }(3) \\
& =((a \vee c) \wedge(b \vee c)) \wedge c=c & \text { by }(4) .
\end{array}
$$

For the second half of (27) compute: $p_{1}=a \wedge c, p_{2}=a \vee c, p_{3}=$ $(b \vee c) \vee c=b \vee c, p_{4}=c$ and so

$$
\begin{aligned}
& p(b, a, b, c, c) \\
= & (((b \vee(a \wedge c)) \vee(a \vee c)) \wedge(b \vee c)) \wedge c \\
= & ((((a \vee b) \vee(a \wedge c)) \vee(a \vee c)) \wedge(b \vee c)) \wedge c \\
& \text { apply }(22) \text { with } x=a, y=b, \text { and } z=c \\
= & (((a \vee(b \vee c)) \vee c) \wedge(b \vee c)) \wedge c \\
& \text { use the second half of (4) with } x= \\
= & a \vee
\end{aligned}
$$

To show the Substitution Property for $\wedge$, let $c \equiv d(\Phi)$ with the sequence $r_{0}, \cdots, r_{n}$ and let $e \in A$. Consider the sequence $e \wedge c=e \wedge r_{0}$, $\left(e \wedge r_{0}\right) \vee\left(e \wedge r_{1}\right), e \wedge r_{1},\left(\left(e \wedge r_{1}\right) \vee\left(e \wedge r_{2}\right), e \wedge r_{2}, \cdots, e \wedge r_{n}=e \wedge d\right.$. For any given $i, 0 \leqq i<n$, either $r_{i} \leqq r_{i+1}$ or $r_{i+1} \leqq r_{i}$. Let us assume that $r_{i} \leqq r_{i+1}$ (if $r_{i+1} \leqq r_{i}$ we proceed similarly). By the definition of $\Phi$, we have $R\left(a, b, r_{i}, r_{i+1}\right)$. By the definition of $R$, this means that

$$
r_{i}=q_{1}\left(a, b, r_{i}, r_{i+1}\right)
$$

and

$$
r_{i+1}=q_{2}\left(a, b, r_{i}, r_{i+1}\right) \text {. }
$$

By (23),

$$
R\left(a, b, q_{1} \wedge e, q_{2} \wedge e\right) \text { and } R\left(a, b, q_{2} \wedge e, q_{1} \wedge e\right),
$$

that is,

$$
R\left(a, b, r_{i} \wedge e, r_{i+1} \wedge e\right) \text { and } R\left(a, b, r_{i+1} \wedge e, r_{i} \wedge e\right) .
$$

Therefore, by the definition of $R$ :

$$
R\left(a, b, r_{i} \wedge e,\left(r_{i} \wedge e\right) \vee\left(r_{i+1} \wedge e\right)\right)
$$

and

$$
R\left(a, b, r_{i+1} \wedge e,\left(r_{i} \wedge e\right) \vee\left(r_{i+1} \wedge e\right)\right),
$$


showing $c \wedge e \equiv d \wedge e(\Phi)$.

Using (24) rather than (23) we prove that $c \vee e \equiv d \vee e(\Phi)$.

Thus $\Phi$ is a congruence relation.

Observe that $p(t, a, b, a, b)=(t \vee a) \wedge b$. Thus $p(a, a, b, a, b)=$ $a$ and $p(b, a, b, a, b)=b$, proving $a \equiv b(\Phi)$.

Finally, if $a \equiv b(\Theta)$ for any congruence relation $\Theta$, then $\Phi \leqq \Theta$, thus $\Phi=\Theta(a, b)$.

Now let $c \equiv d(\Theta(a, b)), c=r_{0}, \cdots, r_{n}=d$ as given in the definition of $\Phi$. For a given $i$, then $r_{i}=q_{1}\left(a, b, r_{i} \wedge r_{i+1}, r_{i} \vee r_{i+1}\right)$ and $r_{i+1}=$ $q_{2}\left(a, b, r_{i} \wedge r_{i+1}, r_{i} \vee r_{i+1}\right)$. Substituting :these into (25) and (26) we obtain the crucial equations:

$$
\begin{aligned}
& a \wedge\left(r_{i} \wedge b\right)=a \wedge\left(r_{i+1} \wedge b\right) \\
& \left(a \vee r_{i}\right) \vee b=\left(a \vee r_{i+1}\right) \vee b
\end{aligned}
$$

for all $i=0, \cdots, n-1$. Thus

$$
a \wedge(c \wedge b)=a \wedge(d \wedge b)
$$

and

$$
(a \vee c) \vee b=(a \vee d) \vee b
$$

In other words, we have shown that $c \equiv d(\Theta(a, b))$ implies the two previous equations, which is the hypothesis of Theorem 2.

Therefore, by Theorem 2, $K \cong Z$. Combining this with $Z \subseteq K$ we conclude that $\boldsymbol{K}=\boldsymbol{Z}$, completing the proof of Theorem 3 .

It should be noted that it is much easier to prove that $Z$ can be characterized by a finite set of identities. The proof given above actually exhibits one such set.

No more than five variables were used in the identities in $\Sigma$, hence,

CoRollary 1. An algebra $\langle A ; \wedge, \vee\rangle$ belongs to $Z$ if and only if every subalgebra of $\langle A ; \wedge, \vee\rangle$ generated by five elements belongs to $Z$.

It is easily seen, that in the Corollary, "five" cannot be replaced by "three". We do not know whether "four" would do.

Since two identities of an idempotent class can always be substituted by one, the finite set $\Sigma$ of Theorem 3 can be reduced to three identities. R. Padmanabhan [15] has shown that the three identities can be replaced by two. 


\section{PART II. Structure Theorems.}

6. Finite algebras. The main result of this section is the following.

THEOREM 4. Every finite algebra $A$ of $Z$ has a representation of the form

$$
A \cong D \times Z^{k},
$$

where $D$ is a (finite) distributive lattice and $k$ a nonnegative integer. In this representation $k$ is unique and $D$ is unique up to isomorphism. In fact, $D$ is a maximal homomorphic image of $A$ in $D$.

This result is based on three lemmas.

LEMMA 8. Let the algebra $A$ be a subdirect product of the algebras $A_{1}, \cdots, A_{n}$. Let us assume that there exists a family $p_{\lambda}, \lambda \in \Lambda$ of polynomials satisfying the following two conditions:

(i) $p_{\lambda}(x, y, y, \cdots, y)=x$ holds in all $A_{i}$;

(ii) for $a, b \in A_{i}$ there exist $a_{1}, a_{2}, \cdots \in A_{i}$ and $\lambda \in \Lambda$ such that $p_{\lambda}\left(a, a_{1}, a_{2}, \cdots\right)=b$.

Let, further, $A$ be a subdirect product of $A_{1}, \cdots, A_{n}$ with the property that for each $i, 1 \leqq i \leqq n$, there is an element $\left\langle c_{1}, \cdots, c_{n}\right\rangle \in$ $A$ such that $\left\langle c_{1}, \cdots, c_{i-1}, a, c_{i+1}, \cdots, c_{n}\right\rangle \in A$ for all $a \in A_{i}$. Then $A$ is the direct product of $A_{1}, \cdots, A_{n}$.

Proof. For $n=1$ the statement is obvious. Let us assume that it has been proved for all $k<n$. Let $A$ and $A_{1}, \cdots, A_{n}$ be given as in the lemma. Let $B$ be the algebra we get from $A$ by omitting the first component of each element of $A$. Obviously, $B$ is a subdirect product of $A_{2}, \cdots, A_{n}$ and this subdirect product satisfies all the hypotheses of Lemma 8 (the element of $B$ chosen for $i, 2 \leqq i \leqq n$ is the element of $A$ chosen for $i$ with its first component omitted). Therefore, by induction hypothesis, $B=A_{2} \times \cdots \times A_{n}$.

It is also clear that $A$ is a subdirect product of $A_{1}$ and $B$, and (using the hypothesis for $A, A_{1}, \cdots, A_{n}$ and $i=1$ ) there is an element $d \in B$ such that $\langle c, d\rangle \in A$ for all $c \in A_{1}$. Now take an arbitrary $\langle a, b\rangle \in$ $A_{1} \times B$. Since $A$ is a subdirect product of $A_{1}$ and $B$, there exist $e \in A_{1}$ such that $\langle e, b\rangle \in A$. By (ii), there exist $\lambda \in \Lambda$ and $a_{1}, a_{2}, \cdots \in$ $A$, such that $p_{\lambda}\left(e, a_{1}, a_{2}, \cdots\right)=a$. Thus, $\langle e, b\rangle,\left\langle a_{1}, d\right\rangle,\left\langle a_{2}, d\right\rangle, \cdots \in A$ and so (using (ii)):

$$
\begin{aligned}
p_{\lambda}\left(\langle e, b\rangle,\left\langle a_{1}, d\right\rangle,\left\langle a_{2}, d\right\rangle, \cdots\right) & =\left\langle p_{\lambda}\left(e, a_{1}, a_{2}, \cdots\right), p_{\lambda}(b, d, d, \cdots)\right\rangle \\
& =\langle a, b\rangle
\end{aligned}
$$


is also in $A$, proving $A=A_{1} \times B$. Thus,

$$
A=A_{1} \times A_{2} \times \cdots \times A_{n},
$$

completing the proof of the lemma.

Lemma 9. Let us assume that for the algebras $A_{1}, \cdots, A_{n}$ the polynomials $p_{\lambda}, \lambda \in \Lambda$ exist satisfying (i) and (ii) of Lemma 8. In addition, let us assume that for each $a, b, c \in A_{i}, b \neq c$ there is a polynomial $g$ satisfying $g(a, b, c)=a$ for which $g(x, y, y)=y$ holds in $A_{1}, \cdots, A_{n}$. Then any subdirect product of $A$ of $A_{1}, \cdots, A_{n}$ is isomorphic to a direct product of some of the $A_{1}, \cdots, A_{n}$.

Proof. Again, we proceed by induction and the case $n=1$ is obvious. For $1 \leqq i \leqq n$, consider the homomorphism $\varphi_{i}: A \rightarrow A^{(i)}$ which is the map omitting the $i$ th component. If, for some $i, \varphi_{i}$ is an isomorphism, then $A$ is isomorphic to a subdirect product of $A_{1}, \cdots$, $A_{i-1}, A_{i+1}, \cdots, A_{n}$, and by the induction hypothesis, the conclusions of Lemma 9 holds for $A$. So we can assume that no $\varphi_{i}$ is an isomorphism.

Now we show that $A, A_{1}, \cdots, A_{n}$ satisfy the conditions of Lemma 8. We have assumed the existence of the $p_{\lambda}, \lambda \in \Lambda$.

Choose an $i, 1 \leqq i \leqq n$. We want to prove that there exists a $\left\langle c_{1}, \cdots, c_{n}\right\rangle \in A$ such that $\left\langle c_{1}, \cdots, c_{i-1}, a, c_{i+1}, \cdots, c_{n}\right\rangle \in A$ for all $a \in A_{i}$.

To simplify the notation let $i=1$. Since $\varphi_{1}$ is not an isomorphism there are elements $\bar{c}, \bar{d} \in A$ such that $\bar{c} \neq \bar{d}$ and $\bar{c} \varphi_{1}=\bar{d} \varphi_{1}$. In other words, $\left\langle c, c_{2}, \cdots, c_{n}\right\rangle,\left\langle d, c_{2}, \cdots, c_{n}\right\rangle \in A$ for some $c_{2} \in A_{2}, \cdots, c_{n} \in A_{n}$ and $c, d \in A_{1}, c \neq d$.

For an arbitrary $a \in A_{1}$, there are $a_{2} \in A_{2}, \cdots, a_{n} \in A_{n}$ such that $\left\langle a, a_{2}, \cdots, a_{n}\right\rangle \in A$, since $A$ is a subdirect product. Choose a polynomial $g$ satisfying $g(a, c, d)=a$ (and, of course, $g(x, y, y)=y)$. Then $g(\langle a$, $\left.\left.a_{2}, \cdots, a_{n}\right\rangle,\left\langle c, c_{2}, \cdots, c_{n}\right\rangle,\left\langle d, c_{2}, \cdots, c_{n}\right\rangle\right)=\left\langle a, c_{2}, \cdots, c_{n}\right\rangle$ is in $A$, verifying the condition. Thus, by Lemma $8, A=A_{1} \times \cdots \times A_{n}$.

Lemma 10. Any finite subdirect power of $Z$ is isomorphic to some direct power of $Z$.

Proof. We shall verify that the hypotheses of Lemmas 8 and 9 are satisfied in $Z$. Let $\Lambda=\{1,2\}, p_{1}=((x \vee y) \vee z) \wedge x$ and $p_{2}=$ $((x \wedge y) \wedge z) \vee x$. It is obvious that (i) of Lemma 8 holds. Let, say, $a=0$. Then $p_{1}(0,0,0)=0, p_{2}(0,2,1)=1$, and $p_{1}(0,1,2)=2$, verifying (ii) of Lemma 8.

We also select $g_{1}=(x \vee y) \wedge z$ and $g_{2}=(x \wedge z) \vee y$. Obviously, $g_{i}(x, y, y)=y, i=1,2$. If $b \neq c$, then $b<c$ or $c<b$. In the first case let $b=0$ and $c=1$; then $g_{1}(a, b, c)=a$ for $a=0,1$ and $g_{2}(a, b, c)=$ 
$a$ for $a=2$. In the second case, $g_{1}(a, b, c)=a$ for $a=2$ and $g_{2}(a, b, c)=$ $a$ for $a=0$ and 1. This completes the proof of Lemma 10.

Now we are ready to prove Theorem 4 . Let $A$ be a finite algebra in $Z$. The only subdirectly irreducible members of $Z$ are $C_{2}$ and $Z$ therefore $A$ is a subdirect product of two algebras $D$ and $E$, where $D$ is a subdirect power of $C_{2}$ and $E$ is a subdirect power of $Z$. Obviously, $D$ is a distributive lattice. By Lemma $10, E=Z^{k}$ for some integer $k$. Thus we have proved that $A$ is (isomorphic to) a subalgebra of $D \times Z^{k}$. We prove that, in fact, $A=D \times Z^{k}$.

Let 1 be the greatest element of $D$ and $a \in E=Z^{k}$. We show that $\langle 1, a\rangle \in A$. Indeed, since $A$ is a subdirect product of $D$ and $E$ there are elements $b \in E$ and $d \in D$ satisfying

$$
\langle 1, b\rangle \text { and }\langle d, a\rangle \in A \text {. }
$$

Define $e \in E$ by the rule:

$$
\begin{array}{lll}
e(i)=a(i) & \text { if } \quad b(i) \leqq a(i) \\
e(i)>b(i) & \text { if } \quad b(i)>a(i) .
\end{array}
$$

Note that $e(i)$, the $i$ th component of $e$, is in $Z$ so the condition $e(i)>$ $b(i)$ uniquely determines $e(i)$. Choose an $f \in D$ such that $\langle f, e\rangle \in A$. Then

$$
(\langle 1, b\rangle \vee\langle f, e\rangle) \vee\langle d, a\rangle \in A
$$

This element is obviously of the form $\langle 1, g\rangle$, and

$$
g(i)=(b(i) \vee e(i)) \vee a(i)=e(i) \vee a(i)=a(i) .
$$

Thus $g=a$, proving $\langle 1, a\rangle \in A$.

Now take an arbitrary $d \in D$ and $a \in E$. Then $\langle d, b\rangle \in A$ for some $b \in E$. For $a, b \in E$ let us construct $e \in E$ as follows:

$$
\begin{aligned}
& e(i)=a(i) \quad \text { if } a(i)=b(i) \\
& e(i) \neq a(i) \text { and } b(i) \text { if } a(i) \neq b(i) .
\end{aligned}
$$

Then $\langle 1, a\rangle$ and $\langle 1, e\rangle \in A$ and so

$$
(\langle d, b\rangle \wedge\langle 1, e\rangle) \wedge\langle 1, a\rangle=\langle d, g\rangle \in A,
$$

where $g(i)=(b(i) \wedge e(i)) \wedge a(i)$. If $a(i)=b(i)$, then $e(i)=a(i)$, and so $g(i)=a(i)$. If $a(i)<b(i)$, then (we are in $Z$ ) $b(i)<e(i)<a(i)$ and so $g(i)=(b(i) \wedge e(i)) \wedge a(i)=b(i) \wedge a(i)=a(i)$. Finally, if $b(i)<a(i)$, then $a(i)<e(i)<b(i)$, hence $g(i)=(b(i) \wedge e(i)) \wedge a(i)=e(i) \wedge a(i)=$ $a(i)$, proving $g=a$ and $\langle d, a\rangle \in A$. This completes the proof of the first part of Theorem 4 .

To prove the uniqueness of $D$ we show that $D$ is a maximal 
homomorphic image of $A$ in $\boldsymbol{D}$. It is obvious that $D$ is a homomorphic image of $A$ in $D$. Let $\Theta$ be an arbitrary congruence relation on $A$ such that $A / \Theta \in D$. By the Corollary to Lemma $1, \Theta=\Phi \times \Theta_{1} \times \cdots \times$ $\Theta_{k}$ and

$$
A / \Theta \cong D / \Theta \times Z_{1} / \Theta_{1} \times \cdots \times Z_{k} / \Theta_{k}, \text { where } Z_{1}=\cdots=Z_{k}=Z .
$$

Since $Z / \Theta_{i} \in D$ only if $\left|Z / \Theta_{i}\right|=1$, we conclude that $A / \Theta \cong D / \Theta$, proving that $D$ is a maximal homomorphic image. This implies the uniqueness of $D$ up to isomorphism. Knowing that $D$ is unique, it obviously follows that $k$ is unique. This concludes the proof of Theorem 4 .

Corollary. The congruence lattice $C(A)$ of any finite algebra $A$ in $\boldsymbol{Z}$ is a finite Boolean lattice.

Proof. Indeed, if $A=D \times Z^{k}$, then $C(A) \cong C(D) \times C(Z)^{k}=C(D) \times$ $C_{2}^{k}$, and $C(D)$ is known to be Boolean.

7. Free algebras. The following results describe the structure of free algebras over $\boldsymbol{Z}$ in terms of the free algebra over $\boldsymbol{D}$.

THEOREM 5. Let $F_{D}(n)$ and $F_{z}(n)$ denote the free algebra on $n$ generators over $\boldsymbol{D}$ and $\boldsymbol{Z}$, respectively. Then

$$
F_{Z}(n) \cong F_{\boldsymbol{D}}(n) \times Z^{k_{n}},
$$

where $k_{n}=3^{n-1}-2^{n}+1$.

Proof $^{2}$. Let $F=F_{z}(n)$ and $D=F_{D}(n)$, and let $X=\left\{x_{1}, \cdots, x_{n}\right\}$ be a set of free generators of $F$. Obviously, $F_{z}(n)$ is a subalgebra of $Z^{3^{n}}$, hence finite. Thus by Theorem 4,

$$
F \cong D \times Z^{k},
$$

for some nonnegative integer $k$. By the corollaries to Lemma 1 and Theorem $4, k$ is the number of congruence relations $\Theta$ of $F$ satisfying $F / \Theta \cong Z$.

Let $\varphi_{1}$ and $\varphi_{2}$ be homomorphisms of $F$ onto $Z$ inducing the same congruence relation $\Theta$. Then $X \varphi_{1}=X \varphi_{2}=Z$ and $\varphi_{i}, i=1,2$, partitions $X$ into $X_{0}^{i}, X_{1}^{i}, X_{2}^{i}$ by setting $X_{j}^{i}=j \varphi_{i}^{-1}$. Since these partitions are the restrictions of the $\Theta$-classes to $X$, they agree. It is easily seen that for $a \in X_{0}^{i}$ and $b \in X_{1}^{i}$ the fact that $a \varphi_{i}<b \varphi_{i}$ is expressed by $a \vee b \equiv b(\Theta)$. Therefore, for some automorphisms $\alpha$ of $Z$, we have $\varphi_{1}=\varphi_{2} \alpha$. Since the converse is obvious, we conclude that $k$ equals

${ }^{2}$ We would like to thank R. Quackenbush for a considerable simplification of the original proof. 
the number of maps of $X$ onto $Z$ up to automorphisms of $Z$, or equivalently, all maps $\varphi$ of $X$ onto $Z$ satisfying $x_{1} \varphi=0$. There are altogether $3^{n-1}$ maps of $\left\{x_{2}, \cdots, x_{n-1}\right\}$ into $Z$. Of these, $2^{n-1}$ does not have 1 in the image and $2^{n-1}$ does not have 2 in the image, the overlap being one map (the constant 0 map). Therefore, $k=3^{n-1}-$ $2 \cdot 2^{n-1}+1=3^{n-1}-2^{n}+1$, as claimed.

We can apply Theorem 5 to describe all finite projective algebras in $\boldsymbol{Z}$.

CoRollary. A finite algebra $A$ is projective in $Z$ if and only if it is isomorphic to some $P \times Z^{k}$ where $k$ is a nonnegative integer and $P$ is projective in $\boldsymbol{D}$.

Remark. By R. Balbes [2] (see also G. Grätzer and B. Wolk [11]) a finite distributive lattice is projective in $\boldsymbol{D}$ if and only if the join of any two meet irreducible elements is again meet irreducible.

Proof. It is well-known that $A$ is projective if and only if it is a retract (idempotent endomorphic image) of a free algebra. Firstly, let $A=P \times Z^{k}$ where $P$ is projective in $D$. Choose an integer $n$ such that $P$ is a retract on $F_{D}(n)$ and $k<k_{n}$. Then, obviously, $A$ is a retract of $F_{Z}(n)$. Conversely, let $A$ be a retract of some $F_{z}(n)$. By Theorem $4, A \cong D \times Z^{k}$. Since $D$ is a retract of $A$, we conclude that $D$ is a retract of $F_{z}(n)$. By the Corollary of Lemma 1, the retraction must collapse all copies of $Z$, hence $P$ is a retract of $F_{D}(n)$, showing that $P$ is projective in $D$. This concludes the proof.

8. Injective algebras. The algebra $I$ of $Z$ is called injective (see, for instance, [8], §13) if for any $A, B \in Z, A$ a subalgebra $B$, any homomorphism $\varphi: A \rightarrow I$ can be extended to a homomorphism of $B$ into $I$.

Theorem 6. $Z$ is injective in $Z$. Any direct power of $Z$ is injective in $Z$ and, therefore, every algebra can be embedded in an injective. An algebra is injective if and only if it is isomorphic to the extension of $Z$ by a complete Boolean algebra.

Proof. Rather than giving a direct proof of these results we shall employ a trick from [14] and then use a result of [3] to get the last statement of Theorem 6 , which implies the other two.

Let us denote by $\hat{Z}$ the algebra $Z$ with three new nullary operations: 0,1 , and 2 . Let $\widehat{Z}$ denote the equational class generated by $\hat{Z}$. Just as in Lemma 2 , every algebra in $\widehat{Z}$ can be embedded in a direct power of $\hat{Z}$.

$\hat{Z}$ is generated by a finite simple algebra $\hat{Z}$ with no subalgebras 
and so by a result of A. Day [3], the injectives in $\hat{\boldsymbol{D}}$ are exactly the algebras $\hat{Z}[B]$ where $B$ is a complete Boolean algebra (for this concept see [7], §22).

Therefore, it suffices to prove the following statement:

An algebra $A$ is injective in $Z$ if and only if 0,1 , and 2 can be interpreted on $A$ so that the resulting algebra $\hat{A}$ belongs to $\hat{Z}$ and $\hat{A}$ is injective in $\hat{Z}$.

Indeed, if $A$ is injective in $Z$, then for some set $J$ there is a homomorphism (in fact, a retraction) $\varphi$ of $Z^{J}$ onto $A$. We can interpret $0,1,2$ on $Z^{J}$ as on $(\hat{Z})^{J}$, and then on $A$ by $0 \varphi, 1 \varphi$, and $2 \varphi$. This makes $\hat{A}$ a homomorphic image of $(\hat{Z})^{J} \in \hat{Z}$, and so $\hat{A} \in \hat{Z}$. Since $\hat{A}$ is a retract of $(\hat{Z})^{J}$, it is injective in $\hat{Z}$. The converse is obvious. This completes the proof of Theorem 6 .

It follows from Theorem 6 and B. Banasehewski [1] that every algebra $A$ in $Z$ has an injective hull uniquely determined up to isomorphism (leaving $A$ fixed).

\section{REFERENCES}

1. B. Banaschewski, Injectivity and essential extensions in equational classes of algebras, Proceedings of the Conference on Universal Algebra, October, 1969, (Queen's University, Kingston, 1970), 131-147.

2. R. Balbes, Projective and injective distributive lattices, Pacific J. Math., 21 (1967), 405-420.

3. A. Day, Injectivity in congruence distributive equational classes, Thesis, McMaster University, 1970.

4. — A note on the congruence extension property, Algebra Universalis, 1 (1971), $234-235$.

5. E. Fried, Tournaments and nonassociative lattices, Ann. Univ. Sci. Budapest Eötvös Sect. Math., 13 (1970), 151-164.

6. E. Fried and G. Grätzer, On some classes of nonassociative lattices, To appear.

7. G. Grätzer, Universal Algebra, The University Series in Higher Mathematics, D. Van Nostrand Co., Princeton, N. J., 1968.

8. — Lattice Theory, First Concepts and Distributive Lattices, W. H. Freeman and Co., San Francisco, Calif., 1971.

9. G. Grätzer and H. Lakser, Identities for equational classes generated by tournaments, Notices Amer. Math. Soc., 18 (1971), 794.

10. G. Grätzer and E. T. Schmidt, Ideals and congruence relations in lattices, Acta Math. Acad. Sci. Hungarí, 9 (1958), 137-175.

11. G. Grätzer and B. Wolk, Finite projective distributive lattices, Canad. Math. Bull., 13 (1970), 139-140.

12. B. Jónsson, Extensions of relational structures, Symposium on the Theory of Models, North-Holland Publ. Co., Amsterdam, 1965, 146-157.

13. , Algebras whose congruence lattices are distributive, Math. Scand., 21 (1967), 110-121.

14. J. Kagan and R. Quackenbush, Self-dual closure algebras, Manuscript.

15. R. Padmanabhan, Equational theory of idempotent algebras, Algebra Universalis, 2 (1972), 57-61.

16. H. L. Skala, Trellis theory, Algebra Universalis, 1 (1971), 218-233.

Received July 19, 1972 and in revised form October 15, 1972. 


\section{PACIFIC JOURNAL OF MATHEMATICS}

\section{EDITORS}

RICHARD ARENS (Managing Editor) University of California

Los Angeles, California 90024

R. A. Beaumon'T

University of Washington Seattle, Washington 98105
J. Dugundj1*

Department of Mathematics University of Southern California Los Angeles, California 90007

D. Gilbarg and J. Milgram Stanford University Stanford, California 94305

\section{ASSOCIATE EDITORS}

E. F. BECKENBACH

B. H. NeUMANN

F. WOLF

K. YOSHIDA

\section{SUPPORTING INSTITUTIONS}

UNIVERSITY OF BRITISH COLUMBIA CALIFORNIA INSTITUTE OF TECHNOLOGY UNIVERSITY OF CALIFORNIA MONTANA STATE UNIVERSITY UNIVERSITY OF NEVADA NEW MEXICO STATE UNIVERSITY OREGON STATE UNIVERSITY UNIVERSITY OF OREGON OSAKA UNIVERSITY

\section{UNIVERSITY OF SOUTHERN CALIFORNIA STANFORD UNIVERSITY UNIVERSITY OF TOKYO UNIVERSITY OF UTAH WASHINGTON STATE UNIVERSITY UNIVERSITY OF WASHINGTON AMERICAN MATHEMATICAL SOCIETY NAVAL WEAPONS CENTER}

* C. R. DePrima California Institute of Technology, Pasadena, CA 91109, will replace J. Dugundji until August 1974. 


\section{Pacific Journal of Mathematics}

\section{Vol. 49, No. $1 \quad$ May, 1973}

A. Bigard, Free lattice-ordered modules ...........................

Richard Bolstein and Warren R. Wogen, Subnormal operators in strictly cyclic

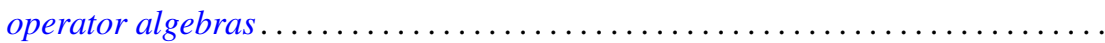

Herbert Busemann and Donald E. Glassco, II, Irreducible sums of simple

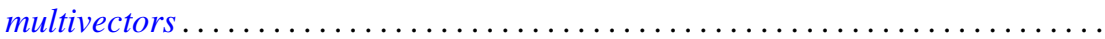

W. Wistar (William) Comfort and Victor Harold Saks, Countably compact groups

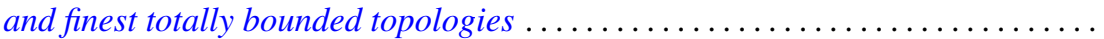

Mary Rodriguez Embry, Maximal invariant subspaces of strictly cyclic operator

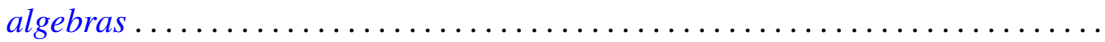

Ralph S. Freese and James Bryant Nation, Congruence lattices of semilattices......

Ervin Fried and George Grätzer, A nonassociative extension of the class of

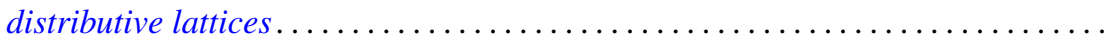

John R. Giles and Donald Otto Koehler, On numerical ranges of elements of locally

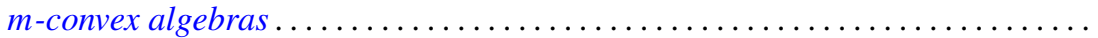

David A. Hill, On dominant and codominant dimension of $\mathrm{QF}-3$ rings ........ John Sollion Hsia and Robert Paul Johnson, Round and Pfister forms over $R(t) \ldots$ I. Martin (Irving) Isaacs, Equally partitioned groups . . . . . . . . . . . . . .

Athanassios G. Kartsatos and Edward Barry Saff, Hyperpolynomial approximation of solutions of nonlinear integro-differential equations.

Shin'ichi Kinoshita, On elementary ideals of $\theta$-curves in the 3-sphere and 2-links in the 4-sphere

Ronald Brian Kirk, Convergence of Baire measures

R. J. Knill, The Seifert and Van Kampen theorem via regular covering spaces ..

Amos A. Kovacs, Homomorphisms of matrix rings into matrix rings ..

Young K. Kwon, HD-minimal but no $H D$-minimal ..........

Makoto Maejima, On the renewal function when some of the mean renewal lifetimes are infinite

Juan José Martínez, Cohomological dimension of discrete modules over profinite groups.

W. K. Nicholson, Semiperfect rings with abelian group of units

Louis Jackson Ratliff, Jr., Three theorems on imbedded prime divisors of principal ideals.

Billy E. Rhoades and Albert Wilansky, Some commutants in $B(c)$ which are almost matrices

John Philip Riley Jr., Cross-sections of decompositions . . .

Keith Duncan Stroyan, A characterization of the Mackey uniformity $m\left(L^{\infty}, L^{1}\right)$ for

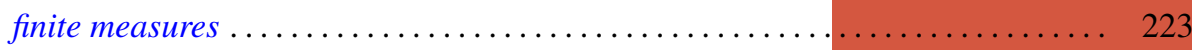

Edward G. Thurber, The Scholz-Brauer problem on addition chains . . . . . . . . . 229

Joze Vrabec, Submanifolds of acyclic 3-manifolds ............

Philip William Walker, Adjoint boundary value problems for compactified singular

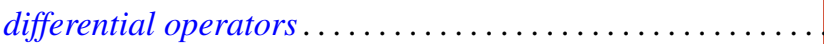

